\title{
A Review On Advanced Applications of BIM in Construction Field
}

\author{
Omkar Patil ${ }^{1 *}$, Umesh Deshpande ${ }^{2}$ \\ ${ }^{I}$ M.Tech. Scholar, Department of Civil Engineering, Govt. College of Engineering, Karad, India \\ ${ }^{2}$ Associate Professor, Department of Applied Mechanics, Govt. College of Engineering, Karad, India \\ *Corresponding author: onkarpatil05@gmail.com
}

\begin{abstract}
The conventional practices being followed in construction industry have lower productivity and lot of delays due to miscommunication and mismanagement between involved parties which are, AECO and investors; which results as loss in economy, time as well as resource wastage. With implementation of 5D BIM, before and during execution phase of construction project, scrupulous management, reduction of the delay in activities as well as wastage of resources; also achieving economy became pragmatic and attainable.

This study focuses on the probable of application areas of BIM technology in construction field. Based on few recent research papers, study aims at exploring the various application areas in BIM technology.
\end{abstract}

Keywords: 5D BIM, Application, BIM, CAD, Civil engineering, Review.

\section{Introduction}

BIM stands for Building Information Modelling. To understand it in simple words, BIM technology is where stakeholders in various disciplines collaborate together and work in synchronize with each other in real time to prepare and share the information necessary. This data and information can be utilized to plan, operate and monitor the ongoing project progress. BIM started getting popular during 1990 and since then it has only evolved to make projects much more standardized.

Few common benefits of BIM are visualization, simulation, cost estimating, construction sequencing, collaboration between multiple stakeholders, better overall design due to overall collaboration, clash detection, Facility management, better service to client and customer, easy to generate fabrication drawings and with 5D BIM we can generate simulation. This study aims at doing literature survey and exploring other application of BIM.

\section{Study of Literature Review for Unconventional Applications of BIM Technology}

A. Xue Li, Jing Xub, Qun Zhang (2017), guides us regarding their major concerns observed such as insufficient planning during initial stage, logical errors in plan, incompetent implementation, inspection, unanticipated duration, cost and quality. Conversely author makes us aware about application advantages of BIM technology such as 3D model, model's integration, model association, management of information, etc. Paper also provides us with BIM application value by referring the report made by Dodge data and analytics, which tracks the impact of technological progress on enterprise via the "smart market". And main findings of it considering Chinese design firms that currently use BIM and not yet applied are BIM application rate is increasing every year, BIM has capability to produce humongous commercial benefits, also currently non BIM using firms are optimistic as $89 \%$ of non BIM users in China were positive about it and none said they are not going to use it at all. Paper explains composition of 5D BIM model, including integrated 5D BIM model, 5D BIM model block. Authors' considers case study of central grand view project as example as to how project works is the city of Zhengzhou City, Qiantang Road and Food Street Intersection Southeast corner. The author finally concludes that the application of BIM technology helps to perform the management of the planning schedule. Author explain us application of BIM with an example like, 3D scanning and printing technology how it can achieve replication and remodeling of building components with an application of energy-saving environmental protection materials. The use of Radio Frequency Identification (RFID) technology in construction projects are helpful in the management of input materials, the control of construction schedules and the management of operation and maintenance.

B. Qiqi Lu, Jongsung Won, Jack C. P. Cheng (2016) claims that previous studies are based on cash outflow instead of cash inflow analysis and project financing. Paper methodology is divided into four steps- 1) BIM model generation, 2) Quantity Take Off extraction, 3) scheduling, 4) cost and schedule loaded with quantity take-off list integration. The paper very well elaborates the methodology with tables of 5D BIM framework. Paper described with two examples that although software like NAVISWORKS, COSTX, Vico-office can also integrate bill of quantity which schedule and Cost data without manual processing, they cannot accurately in analyze cash flow of an AEC project, as software assume that cash flows are continuous with respect to completion of activities but in actual practice there are many activities and tasks where personnel has to pay before actually availing the services, while 
many are realize after some period of activity completion. Also this software cannot give BIM based financial decision making for project financing and hence Framework proposed by paper give more accurate solutions by calculating cash inflows based on contract with clients, the actual cash outflow by considering down payment, payment period and lead time. But author also makes us aware of limitations of this paper which are 1) Framework did not consider stochastic expect to analyze cash flow, 2) Framework does not consider sub-contractor in analyzing cash flows a project, which should be subcontractor's cost accounts for a large portion of general contractor's cost. Paper resumes borrowing money is only financial method and unit price contract in the case study, but all about limitations will be resolved as above are authors' future work.

C. Ing. Radan Tomek, Ing. arch. Sergey Kalinichuk (2015) introduces the synthesis of agile project management and building information modelling in the construction Industry and its application with importance on economic incentives of their implementation as the most significant factor. Agile project management is basically an iterative approach to planning and guiding project processes, the project is segregated into relatively short pieces that are completed in work sessions that run from design phase to testing and quality assurance. These sessions are known sprints and can be a few days or weeks. In the popular and specific Agile development method term scrum is used to represent iteration. The paper takes benefit of APM and BIM having few common Tools and techniques. These tools are here grouped in two broad interrelated categoriesorganizational and Technological. Organizational toolsprovide framework for peoples and machines to work together in sync. Technological tools- facilitate current working within organization. This paper also have well illustration of common goals of BIM and agile. The paper elaborate synthesis of two approaches and comparative advantages of BIM with tables. The paper discusses with us about baseline schedule development approach and a probable estimation of changes connected with hybrid scheduling approach with graphs. It is predicted that integrated product and process model will help in improvement in construction process with respect to: collaborative design, decrease in project duration, decrease in cost, decrease in claims and disputes, increase in product quality. Author supports his claims by giving us examples of group of Russian companies which have already implemented the synthesis of agile and BIM Has proved its applicability and effectiveness.

D. Gaurav S. Narlawar, N. B. Chaphalkar, Sayali Sandbhor (2019) aims to provide a review on applications of primavera software for the time and resource management of construction project. The study explores that Primavera P6 provides solution to various complexities associated with planning, scheduling, controlling, monitoring and tracking of construction, and this observation is based on the literature survey performed by author. The author discussed various obstacles of efficient planning while performing The traditional approach of scheduling according to Oracle, $90 \%$ of project exceed allocated budget so the failure rates of construction project are high. Author put forth various approaches used by cited authors and their results and gets the conclusion that while comparing Primavera P6 and Primavera P6 WebLogic, primavera P6 is less effective in project planning, scheduling and tracing than Oracle primavera P6 WebLogic. But Prima Vera P6 can increase facility distribution by providing detailed information on time management, Cost management, update and monitoring, etc. It is observed that organization can increase profitability and productivity by optimizing Project resources. Although author also elaborates limitation of Primavera software, which are sometimes impractical schedule when ill sequenced progress occurs and results in an information with logical errors and artificially delayed project. Author emphasized that $\mathrm{P} 6$ is better and provide more features essential than MSP 2007 for open work bench 1.1.6.

E. Burcin Becerik-Gerber, Farrokh Jazizadeh, Nan Li, and Gulben Calis (2012) highlights that as FM has activities involving multiple disciplines and it has extensive information required. Some of these needs are mentioned by several existing FM information systems. BIM, holds possibilities for assisting, doing provision and supporting FM practices with its features like that of visualization, analysis and control. This paper explores how BIM can be profit resulting platform for supplementing FM practices. The aim of author is 1) to explore ongoing status of BIM implementation in FM, 2) set examples of BIM implementation and use of FM functions and 3) to provide a data and process required for resource planning. The study claims that BIM implementation is in still operations and maintenance stage, there is limited information on this topic. So authors used few research method which are developed on each other 1) personal interview- were conducted to examine FM functions and processes how BIM could improve personal and process efficiency, 2) online survey- on the basis of analysis of personal interviews, authors constructed more structured questionnaire to understand industry needs and how BIM could be implemented to FM field at process level, 3) expert interview- a significant amount of information was gathered from face to face and on the phone interview were conducted with 22 industry professionals. After that others experiences with chart regarding profiles of respondent organizations, functions of organizations of respondents, project stages that BIM is currently used for, what our future plans of implementation of BIM in Nan-BIM organizations, and potential FM application areas that BIM can be used for. This study also Well elaborates potential application areas where BIM can be used for which are locating building components, providing real-instance data access, visualization and marketing, checking whether project could be maintained at ease, creating digital assets, space management, planning and feasibility studies for the projects with not much initial funding construction, emergency management, controlling and monitoring energy, personnel training. Also it mentions how 
much percentage of BIM and non-BIM you just have been asked and it is observed that BIM users were more optimistic than non-BIM users.

F. Shu-Hui Jan, Shih-Ping Ho, Hui-Ping Tserng (2013) guides us in the phenomenon of knowledge management. KM is gathering of procedures which control the generation, storage, reuse, assessment and experience based knowledge in a typical situation of problem solving contest. This study give emphasis on the sharing of knowledge between stakeholders and its benefits which could be achieved such as decreasing wastage of time and cost of finding quick solutions to problems related to situation whether construction is feasible, also sharing the information is key stone in avoiding the mistakes that have been observed in the projects before. Also knowledge and experience sharing reduces need of finding solutions to the problems from the start. Paper proposes new methodology to provide sharing of construction knowledge by using BIM approach, during the working stage of construction and put forth BIM based knowledge sharing management system for project managers and engineers. This system was applied in a case study of construction project in Taiwan to verify the mentioned methodology and efficacy of sharing environment. Combined results observed in case study show us that BIMKSM can be used as Visual BIM based KSM platform with use of BIM approach and Web Technology. Case study was in Taiwan and BIMKSM utilization what done in construction project, case study was implemented in mid part of construction phase, and it was undertaken during 4-month construction project. Although some limitation were encountered, which might have been resolved in future scope which are 1) untimely and tiny updated information related to different types of knowledge, 2) high time and assistance needed for engineer and managers to use BIM software to edit and update knowledge feedback, 3) non enthusiast of participants which do not share their own knowledge and experience without enough incentive. According to author, field results suggest that BIMKSM system is an efficient and easy to use platform for knowledge management in construction project. Results of case study demonstrate the efficacy of BIMKSM like knowledge management system due to its inclusion of BIM and web technologies during working phase. In summary, engineers were able to improve and enlarge their understanding of previous grasped knowledge and experience from all participants.

G. Abdulsame Fazli, Sajad Fathi, Mohammad Hadi Enferadi, Mayram Fazli, Behrooz Fathi (2014) focuses on Iranian construction industry. Authors' claim that Iranian construction is getting criticized that its efficacy is low. It is claimed that 80 percent data within the building process is the identical for almost all the projects and hence there are humongous opportunities for improvisation. The purpose of this paper is to analyze how project managers can use BIM (Building Information Modeling) as a good tool for simulating project condition in order to avoid rework and wasting time and costs.
It was concluded that project managers in general, do lack the knowledge regarding and application BIM which make them less efficient. The author emphasizes that human beings are irreplaceable even after using BIM. BIM reduces unnecessary and monotonous work, and helps in data processing. Another common mistake is that people assume that BIM doesn't have any errors at all, while that's not all true. Since humans may unknowingly insert wrong data, the errors in BIM are may occur. In this study, 30 projects in the world that have used BIM as have been reviewed to assess the outcome of using BIM in construction projects. The source was case studies is the internet. It is noted that the Success Criterion for Coordination was created from the PMBOK Knowledge Area of Integration Management. These works also concluded a new point of view for handling data, shifting from the document pattern to the paradigm of the Project Integrated Database. The research study done on that basis has shown that it does, with Communication and Coordination as two of the Key Performance Indicators (KPIs) showing more benefits due to BIM's use in construction projects. Author with use of two well explained tables provide us with probable benefit of using BIM for project managers based on PMBOK knowledge areas. Authors' claimed that in contrast to traditional projects in which model visualizations had to be made from start, visualizations may be made from previously created models in projects that use BIM. In BIM, the basis for decision-making is better than in traditional projects. At the end, authors' concludes that it is easy to observe the results of decisions when using BIM in real time. The project managers interviewed predicted possible improvements out of linking the building information model to time, which would lead to better control during the working phase. Almost half of the respondents were of the opinion that the Project Manager should be BIM Professional and 24 percent were of the opinion that the Project Manager should be in charge of overall BIM Management.

H. Peter Smith (2014) examines global issues related to the role of project cost management professionals in the implementation and development of Building Information Modeling (BIM) in the construction industry. The paper is based on an evaluation of ongoing industry trends and affairs linked to the enactment of BIM and in detail interviews with quantity survey organizations in Australia. This paper explores the importance for project cost management professionals to be fully involved in all phases of the project and to employ the 5th dimension of becoming important phase in the BIM environment - the '5D Project Cost Manager.' The paper concludes with the findings that it is highly important to be project manager who knows the phenomenon and application of 5D BIM and to be able to take off schedules for the virtual 5D models and living cost plans in real time. The literature survey done claimed that no study currently has been done on the level of BIM acceptance and enactment of the quantity surveying profession in Australia. The research methodology adopted for the study was to perform industry interviews with 
medium to large quantity survey firms in Australia. The number of surveying firms consisted of three medium-sized enterprises (ten to twenty employees) and 3 big firms (twenty plus employees). All of the firms had offices in Australia, but the concentration was kept on the quantity of surveying services provided by the home office. The companies were located in NSW and Queensland. Results of the research clarify that the quality of model generated depends upon the complexity of the model, which hindered by insufficient consultancy fees provided by client. Moreover, regarding the automated quantity take-off, according to many firms nothing has changed and they still need to check about inaccuracy and noise in output data. Quality of electronic documentation is still measure concern. Authors' also mention that new generation of quantity surveyors will emerge which may become overly dependent upon automated 3D generated documentation, and as they lack the 2D paper based measurement, may encounter difficulty in finding the errors in data. Authors' emphasize that there is still void in the standardization and software compatibility issues between multiple platforms. Although they are optimistic that this may resolve in future updates. Firms agreed that cost data sharing with multiple stakeholders will be accepted as BIM becomes more mainstream over time, either share data or not. Insecurity over legal liability is also creating problems for insurance companies in the industry which have obvious implications for firms providing services to BIM projects. At the end, authors' come to conclusion that although old firms may find it as threat but BIM should be perceived as opportunity, as it eases the overall documentation, visualization and time effective.

I. Saeed Rokooei (2015) has the aim of paper to show the sharing between BIM and the role of project managers in construction projects. Study highlights the importance of having the appropriate BIM knowledge and experience for project managers to completion of project. The study also discusses the requirements for BIM knowledge and experience in the improvisation of project managers. Author introduces us with BIM, its history, role of BIM in construction project management and its use as toll for project managers. Author puts light on aspects of BIM which are integrated project delivery, which is an increasing approach to project delivery that combines and integrates all stakeholders, project managers, engineers, designer, systems and procedures being followed into a collaborative process. IPD optimizes the value of the project by improving efficiency in all phases. Moreover, author give emphasis on the efforts put by many BIM development firms such as Building SMART, which developed an Industry Foundation Classes (IFCs) which are file format, which is supported by all BIM software, which enables users having multiple BIM platforms to operates inter connectively. Author further mentions technical aspects of BIM like Clash detection means it highlights elements in model when they are overlapped between different disciplines, constructability useful for risk mitigation, Analysis which helps PM in decision making, time
(4D) and cost (5D) estimation which basically drive the overall project, Integration between personnel of multiple disciplines, Quantity take-off, Collaboration between teams of various disciplines, Communication which is very vital for smooth progression of any project. While explaining the role of BIMM in project management, author believes that BIM and PMBOK has many things in common which makes BIM a project management toll such as Integration management, BIM is object based environment that can categorize different elements of model in different groups., this concept occurs in project scope management of PMBOK. Another is managing the time and cost which are already an important concept in BIM. While collaboration and team building is what HRM considers under project management areas and communication in main frame of BIM. Clash detection works like quality control of the project mentioned in PMBOK. Author highlights that proper project manager's education and knowledge are very important while working with BIM, as characteristics of user to the great extent affects the overall output and quality also accuracy of the BIM model and its results. So, author states that project manager should be one of the best user, while working with the BIM.

J. Ireneusz Czmoch, Adam Pekala (2014) shortly present the history and development of traditional civil engineering design. Next, the idea of Building Information Modeling (BIM) and its pragmatic advantages are explained. The main part of the paper is concentrated on discussing what type of difficulties we may come across during the enactment of BIM technology and how they relate to probable benefits. Authors' mention benefits of $\mathrm{BIM}$, collision detection, various dimension dedicated to particular aspect of project planning (4D-time, 5D- cost, 6Dsustainability, 7D-facility management application), etc. Another benefit is that structural design suitable for given architectural plan and elevation of building can be efficiently found using BIM than traditional approach. Case study chosen was office complex Warsaw, 14 storey building, Revit was used to prepare BIM model and 10 designers were simultaneously working on it, still results obtained were $10 \%$ faster and $80 \%$ accurate as the authors' claims. Collision were detected and early resolved, model was set to be collaborated with multiple discipline platforms and rate of synchronization was 30 Minutes each. Author talks about BIM drawbacks as followsHigh implementation cost, education cost of BIM modeler, Accuracy of the model depends upon fluency and experience of the modeler, it is necessary for users to constantly stay updated with new emerging nomenclature, phrases, vast array of latest expression, moreover it is also worth noting that a full BIM model is only useful, also there is great uncertainty amongst the involved parties regarding who should bear the cost of BIMinvestor, consultancy or client, also there is great uncertainty regarding lack of legal regulation. Author at the end concludes that as more people get involved in BIM technology, BIM can become pragmatic for even small to medium firms. 


\section{Conclusion}

From above literature survey, it is observed that BIM possess tremendous potential to revolutionize the construction industry in much organized, fast paced, better collaborated, well qualitative output and better managed direction. With BIM technology, sharing of knowledge, also problems are possible in real time and so the solutions. It is understood from the survey that construction industry is still hesitating, if not avoiding the implementation BIM. But, as this industry is dynamic and understands the value and need of time, it will in near future implement the BIM and make industrial output in standardized format. Although BIM technology has some limitations like legal documentation issues, initial high cost, need of proficient personnel to operate the software and similar barriers, its benefits far exceed the limitations and is definitely the path setter in construction industry. So, for large scale project, BIM implementation is recommended.

\section{References}

[1] Xue Li, Jing Xub, Qun Zhang, "Research on Construction Schedule Management Based on BIM Technology," Procedia engineering, vol. 174, pp. 657-667, Mar. 2017.
[2] B. Qiqi Lu, Jongsung Won, Jack C. P. Cheng, "A financial decision making framework for construction projects based on 5D Building Information Modeling (BIM)," International Journal of Project Management, vol. 34, no. 1, pp. 3-21, Jan. 2016.

[3] Radan Tomek, and Sergey Kalinichuk, "Agile PM and BIM: A Hybrid Scheduling Approach for a Technological Construction Project," Procedia engineering, vol. 123, pp. 557-564, Oct. 2015.

[4] Gaurav S. Narlawar, N. B. Chaphalkar, Sayali Sandbhor, "Application of Primavera Software in Management of Construction Project: A Review," international journal of scientific \& technology research, vol. 8, no. 8, Aug. 2019.

[5] Burcin Becerik-Gerber, Farrokh Jazizadeh, Nan Li, and Gulben Calis, "Application Areas and Data Requirements for BIM-Enabled Facilities Management," Journal of Construction Engineering and Management, vol. 138, no. 3, Mar. 2012.

[6] Shu-Hui Jan, Shih-Ping Ho, Hui-Ping Tserng, "Applications of Building Information Modeling (BIM) in Knowledge Sharing and Management in Construction," World Academy of Science, Engineering and Technology, International Journal of Civil and Environmental Engineering, vol. 7, no. $11,2013$.

[7] Abdulsame Fazli, Sajad Fathi, Mohammad Hadi Enferadi, Mayram Fazli, Behrooz Fathi, "Appraising effectiveness of Building Information Management (BIM) in project management," in CENTERIS 2014 Conference on Enterprise Information Systems, Procedia Technology 16, pp. 1116-1125, 2014.

[8] Peter Smith, "BIM \& the 5D Project Cost Manager," Procedia - Social and Behavioral Sciences, vol. 119, pp. 475-484, Mar. 2014.

[9] Saeed Rokooei, "Building Information Modeling in Project Management: Necessities, Challenges and Outcomes," Procedia - Social and Behavioral Sciences, vol. 210, pp. 87-95, Dec. 2015. 